\title{
PERFORMANCE EVALUATION OF ROUTING ALGORITHM FOR MANET BASED ON THE MACHINE LEARNING TECHNIQUES
}

\author{
Dr. M. Duraipandian, \\ HoD/CSE, \\ Vivekanandha College of technology for women. \\ Namakkal. \\ svsduraipandian@gmail.com
}

\begin{abstract}
The rapid advances in wireless communication technology has led to an extraordinary progress in the adhoc type of networking. The mobile adhoc networks being a subtype of the adhoc network almost poses the same characteristics of the adhoc network, presenting multiple challenges in framing a route for the transmission of the information from the source to the destination. So the paper proposes a routing method developed based on the reinforcement learning, exploiting the node information's to establish a route that is short and stable. The proposed method scopes to minimize the energy consumption, transmission delay, and improve the delivery ratio of the packets, enhancing the throughput. The efficiency of the proposed method is determined by validating its performance in the network simulator-II, in terms of the energy consumption, delay in the transmission and the packet delivery ratio.
\end{abstract}

Keywords: wireless communication, Adhoc networks, MANET, routing protocols, reinforcement learning, energy consumption, transmission delay and packet delivery ratio

\section{Introduction}

The progressive developments in the wireless communication has mad the mobile adhoc networks prominent among a wide range of applications[3] The mobile or the wireless adhoc network is a frame work utilizing the nodes or the devices that are mobile to develop a potent network that does not rely on any infrastructure, [2].The nodes incorporated into this mobile or the wireless adhoc network are never stationary, they roam freely and extend communication with the nearby device over the wireless medium, without a need for a central body to control their actions. The nodes being mobile shit their places regularly and rapidly and configure themselves swiftly [9] .The nodes in the adhoc network take up the role of the hosts as well as the routers ensuring the delivery of the data, [2], so due to their exclusive characteristics the mobile adhoc networks present multiple challenges in routing. So the routing algorithm takes the significant part in the architecture design of the MANET, [3] these unique characteristics make them a prominent in a wide range of application, some of the applications of the MANET are military reign, disaster areas, oceanography, medical fields, commercial services, personal area network, rescue missions etc. The necessary steps in forming an efficient routing methodology over coming all the crucial challenges presented by the mobile adhoc 
Journal of trends in Computer Science and Smart technology (TCSST) (2019)

Vol.01/No.01

Pages: 24-35

https://irojournals.com/tcsst/

DOI: https://doi.org/10.36548/jtcsst.2019.1.003

network led to many explorations that lead to the development of the routing facilities, some of the convention used routing methodologies for the adhoc network were table driven, on demand, location based, hierarchical, hybrid and the multipath [1], taking the concepts of the conventional methods as base many ideas were developed enhancing and the modifying the conventional methodologies of routing available for the adhoc networks, [10], [11], [12] . To reduce or improvise the quality of service of the mobile adhoc networks, in its energy utilization as the nodes involved are low power devices, thereby in return enhancing the life of the network. To improve the latency in the transmission by establishing a shortest path. Improving the routing facility that generates an adaptive greeting message that is broadcasted periodically along with the nodes speed to reduce the congestion and the overhead in the network [5] the routing hybridizing two or multiple methods were also exploited [13], [14], [15] in finding a more convenient method, to minimize the end to end delay and the memory and the power consumption. The fig.1 below gives the some of the routing methods available for the MANET

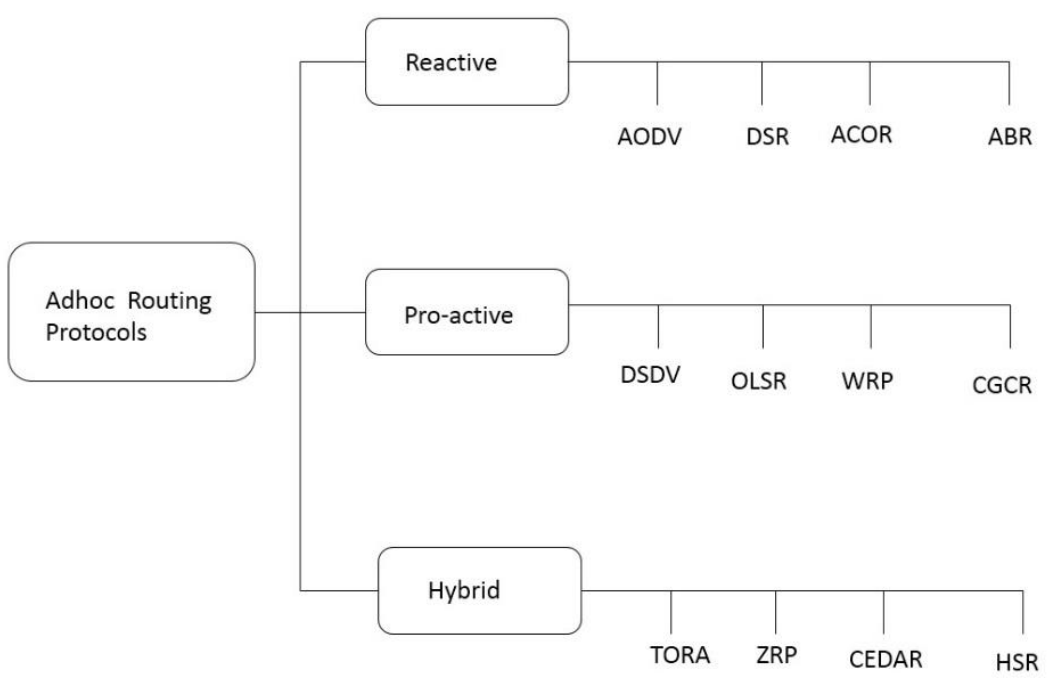

Fig .1 Routing Protocols for MANET

All the methods explored so far exploited methods to improve the network life by reducing the energy consumption and reducing delay exploring a shortest path for the data transmission, but failed to concentrate in retaining the stable path to have a continuous communication.

So the proposed method utilize the type of the machine learning, the reinforcement learning for mobility prediction of nodes in relation to the destination exploiting the information nodes that could aid in establishing the shortest route along with the stable path. 
Journal of trends in Computer Science and Smart technology (TCSST) (2019)

Vol.01/No.01

Pages: $24-35$

https://irojournals.com/tcsst/

DOI: https://doi.org/10.36548/jtcsst.2019.1.003

The remaining paper is organized with the 2 . The related works on the routing protocols for the mobile adhoc networks, 3. The proposed work, exploit reinforcement learning in identifying a short and a stable path, 4. The performance evaluation of the proposed method in network simulator-II, 5. Conclusion.

\section{Related works}

Saeed et al [1] presents the classification of the routing protocols of the mobile adhoc network based on their characteristics and the design doctrine of the routing methods or the network frame work. Bai et al [2] presents the performance evaluation of the two flat routing protocol proactive and the reactive routing protocols along with the simulation result justifying their performance Nayak et al [3] the author proposes the routing considering the mobility of the nodes, and evaluates the reactive routing under different mobility to improve the applications of the protocol. Er-Rouidi, et al [4] the paper elaborates the energy consumption of the routing protocols, by comparing the performance of the four routing protocols DSR, OLSR, DSDV and the AODV , further evaluates the parameters of the network that influence's the energy consumption in the various routing protocols under variety of traffic and the mobility models. Darabkh, et al [5] the literature presents the dual phase AODV that is also mobility aware to establish more stable routes, diminishing the route failures, Majd, et al [6] the author evaluates the network performance of the MANET in the terms of the energy usage, routing overhead, throughput and delay comparing the performance of the various routing protocols that come under the table driven and on demand. Ghouti et al [7] the author utilizes the extreme machine learning to design a mobile adhoc network that is enriched with the prediction of its mobility, but does pay heed to the prediction accuracy limitations while calculating the distance between the neighboring nodes. Forster et al [8] the survey on the machine learning techniques for the adhoc networks classifying the available methods and evaluating them and presenting the most applicable algorithms suited for the adhoc networks Darwish et al [9] the paper presents the firefly algorithm in finding the shortest optimal path for the routing in the wireless adhoc network. Li, et al [10] paper utilizes the reinforcement learning in the VANET to deliver the information with the limited amount of delay and hops. Ghasemnezhad et al [11] the author proposes a reliable and a secure routing for the identifying the shortest path for the adhoc networks using the fuzzy based logic Thangaramya et al [12] presents the CNN trained fuzzy network to frame the shortest routing for the wireless sensor network that serves as the underlie for the data procurement in the internet of things. Nallusamy, et al [13] the author presents the particle swarm optimization based geographical routing, overcoming the demerits of the geographical based routing in improving the network lifetime. Kwon et al [14] the paper exploits the BDT and the RODT in forming a traffic aware multipath routing to reduce the overhead caused in the routing. Sajwan et al [15] the author presenting the novel algorithm utilizing both the flat and the hierarchical routing in order to improvise the energy efficiency

\section{Proposed Work}

ISSN: 2582-4104 (online) 
Journal of trends in Computer Science and Smart technology (TCSST) (2019)

Vol.01/No.01

Pages: 24-35

https://irojournals.com/tcsst/

DOI: https://doi.org/10.36548/jtcsst.2019.1.003

The disputes faced in the mobile adhoc networks in the routing of information from the source to the destination is addressed in the proposed work, the for each node from the set of nodes $(N D)$, where $N D=\left\{n_{1}, n_{2} \ldots \ldots \ldots n_{i}\right\}$, the information of its availability in relation to the destination is gathered, in order to establish a stable short path between the source to the destination. The proposed work utilizes the reinforcement learning to identify the short stable path in the mobile adhoc network to improve the delivery ratio of the information, thereby increasing the throughput and e minimizing the energy consumption and the delay, by the founding a short trail for the conveyance.

The paper explores the mobility pattern of the nodes by the reinforcement learning to have more optimal trail for the transmission of the information's or the data packets from the source to destination.

\subsection{Exploration of Reinforcement Learning}

Reinforcement learning, supervised and the unsupervised are the three basic machine learning structure, in which the reinforcement learning is based on the concept of the interacting, where the learning is done by the conversation or the interaction extended between the learner $(l)$, the deciding authority $\left(D_{A}\right)$ and the surrounding system or environment . Here the decision maker and the learner are called the agent. Each time the learner and the decision maker proceeds with the certain actions the surrounding reacts back to it, that is it provides with the reward that might be positive or negative. The actions performed by the learner and the deciding authority, takes them to a new level or a position often indicated as the state $\left(S_{T}\right)$. The reinforcement learning is modelled based on the Markov Decision Process, but do not consider the actual mathematical frame work of the Markov Decision Process, with the set of environment $(E N)$ and the states of agent $S_{T}$, and with a certain set of action $\left(A_{S}\right)$ performed by the agents. The probability of the agent getting shifted from its present state $\left(S_{T}\right)$ to a new state $\left(\left(\bar{S}_{T}\right)\right.$ is estimated as shown in the equation (1)

$$
\operatorname{Prob}_{A_{s}}=\operatorname{prob}\left\{S_{t+1}=\bar{S}_{T} \mid S_{t}=S_{T}, A_{t}=A_{s}\right.
$$

Where ' $t$ ' represents the time of the occurrence of the action. Once the $l$ and the $D_{A}$ shift from one state to the other after completion of an action, the $E N$ presents with a reward $\left(R_{A_{S}}\right)$. The equation (2) is framed in this regard.

$$
R_{A_{s}}=E\left\{r e_{t+1} \mid S_{t}=S_{T}, A_{t}=A_{s} S_{t+1}=\bar{S}_{T}\right.
$$

The value function defined for a state, determines the quality of the state in relation to the $l$ and $D_{A}$, the value function in respect to the particular policies is given in equation (3) 
Journal of trends in Computer Science and Smart technology (TCSST) (2019)

Vol.01/No.01

Pages: 24-35

https://irojournals.com/tcsst/

DOI: https://doi.org/10.36548/jtcsst.2019.1.003

$$
\text { value }_{\text {func }}=E\left\{\text { re }_{t} \mid S_{t}=S \Rightarrow \sum_{A_{S}} \pi\left(s, A_{S}\right) \sum_{\bar{S}_{T}} \operatorname{Prob}_{A_{S}}\left[R_{A_{S}}+\text { vvalue }_{\text {func }}\left(\bar{S}_{T}\right)\right.\right.
$$

Where, $\vartheta$ represents the values of the states that influence the current state $(0>\vartheta>1)$. The value functions based on the action represents the total reward for a particular action and the policy $(\pi)$ is used from the proceeding state up to the final state and the $\pi\left(S_{t}\right) \rightarrow A_{s}$ is the policy function. The optimal value function for a particular action that does not depend on the policy is expressed as in the equation (4)

$$
\text { op }\left(\text { value }_{\text {func }}\right)_{A_{S}}=\sum_{\bar{S}_{T}} \operatorname{Prob}_{A_{S}}\left(R_{A_{S}}+\vartheta \max \text { op }\left(\text { value }_{\text {func }}\right) \bar{A}_{S}\right.
$$

The equations (4) opts for the action with the heightened value.

\subsection{Reinforcement Learning for Routing in Mobile Adhoc Network}

The routing being the crucial part in the mobile adhoc network, the proposed method utilizes the reinforcement learning in the routing of the packets from the source to the destination. The reinforcement learning selects the nodes based on the values of the actions, where the actions include the determination of the delay that is acquired from the nodes available along with the successful transmissions of the each node. The nodes determined with minimum delay and the maximum successful transmission is framed into a group using the k- means clustering. The optimal group from the number of groups available is selected based on its reward. The optimal group selection is followed by the selecting of the neighboring node for the next hop, the node with the minimum distance to the destination is opted to be the next hop, and the equation (5) gives the policy used in selecting the group.

$$
\pi\left(S_{T}, A_{S}\right)=\frac{e^{\frac{o p\left(\text { value }_{\text {func }} A_{S}\right.}{t}}}{\sum_{A_{S}} e^{\frac{o p\left(\text { value }_{\text {func }} A_{S}\right.}{t}}}
$$

So here the actions refers to the group, and the value obtained for the groups are based on the rewards of the nodes, further the shortest path is enumerated by determining the nodes with the minimum distance from the destination. The equation (6) and (7) gives the value estimation of the groups, based on the delay of the transmission (delay $\left.y_{\text {trans }}\right)$ and the number of successful transmission $\left(\operatorname{succ}_{\text {trans }}\right)$.

$$
\text { delay }_{\text {trans }}=A_{s} *\left(r e_{t}+(\vartheta * \text { MINvalue })\right)
$$


Journal of trends in Computer Science and Smart technology (TCSST) (2019)

Vol.01/No.01

Pages: 24-35

https://irojournals.com/tcsst/

DOI: https://doi.org/10.36548/jtcsst.2019.1.003

$$
\operatorname{succ}_{\text {trans }}=A_{s} *\left(r e_{t}+(\vartheta * \text { MAXvalue })\right)
$$

The algorithm given below in fig .2 explains the steps involved in the neighbor node discovery and the shortest path selection.

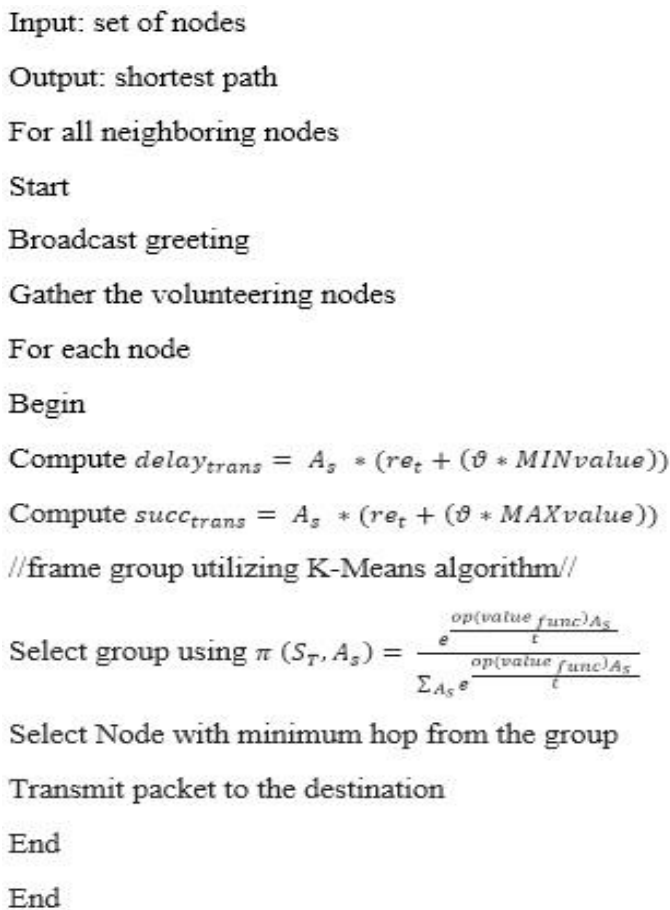

Fig.2 Algorithm for short Path Selection

Though the algorithm enables us to identify the shortest path this becomes unsuitable when the nodes wander from its initial position. So the in the proposed process the reinforcement is also utilized in estimating the mobility patterns of the node to analyze behavior of the nodes.

\subsection{The Mobility Pattern Prediction of the Mobile Nodes}

The mobile nature of the nodes is also a major issue that affects the routing in the networks. In order to predict the current location of the mobile nodes. The proposed routing structure frames multiple states, the mobility behavior causing the node to shit form one position to the other could be predicted, using the states. The following table.1 gives the different states involved in determining the mobility pattern of the nodes. 
Journal of trends in Computer Science and Smart technology (TCSST) (2019)

Vol.01/No.01

Pages: $24-35$

https://irojournals.com/tcsst/

DOI: https://doi.org/10.36548/jtcsst.2019.1.003

\begin{tabular}{|l|l|}
\hline State 1 & $\begin{array}{l}\text { Determines whether packet could be transmitted directly or through route } \\
\text { discovery }\end{array}$ \\
\hline State 2 & Initiates the route discovery operation \\
\hline State 3 & Conducts Route Discovery, waits for inputs signal for making decisions \\
\hline State 4 & Performs routing operation, \\
\hline State 5 & $\begin{array}{l}\text { Gathers reply and updates the table containing routing information goes to } \\
\text { State } 4 \text { for performing routing }\end{array}$ \\
\hline State 6 & Operates as interface node transmitting packet to the Destination. \\
\hline State 7 & Accepts acknowledgement and performs necessary value updates \\
\hline State 8 & Determines the self- interrupt processing state \\
\hline State 9 & Continues with transmission and proceeds to state 1 for next transmission \\
\hline
\end{tabular}

Table .1 States for Mobility Prediction

Whenever a packet has to be transmitted from the node to the destination, the nodes in spite of its mobile nature is assumed to found in any one of the above states mentioned and acts accordingly, this ensure the stability of the path, during the transmission thus reducing the link failures, and increasing the throughput of the network, the flow diagram given below in the Fig. 3 shows the method of mobility pattern prediction done in the proposed method.

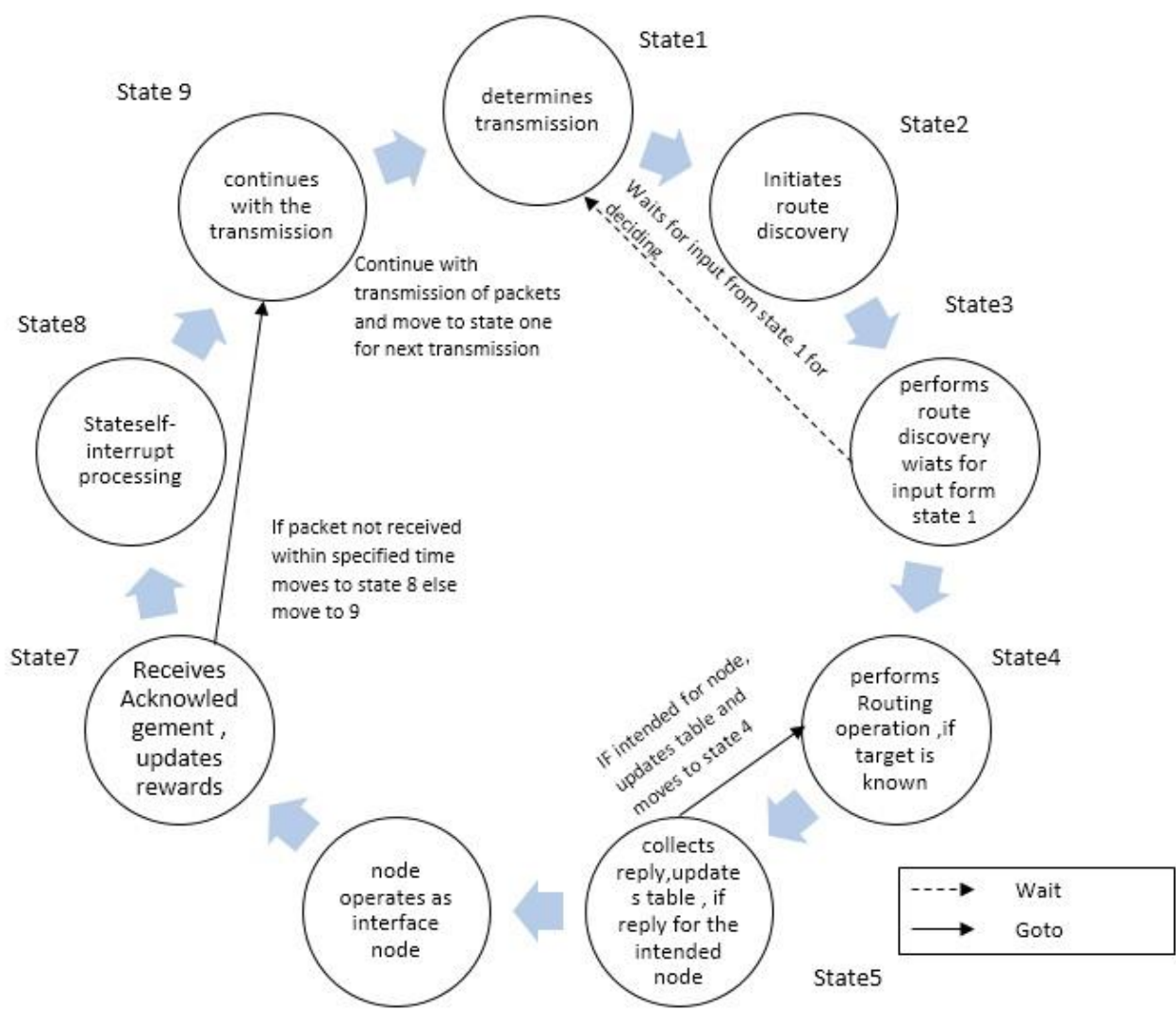

State 6 
Journal of trends in Computer Science and Smart technology (TCSST) (2019)

Vol.01/No.01

Pages: 24-35

https://irojournals.com/tcsst/

DOI: https://doi.org/10.36548/jtcsst.2019.1.003

Fig .3 Proposed Flow Diagram for Mobility Prediction

\section{Result and Discussion}

The proposed method evaluated with the network simulator-II tool to evince its efficiency, in return to the stable short path established by the proposed method. The proposed method paving a stable route for the mobile adhoc network, is evaluated with the varying number of nodes ranging from the 100 to 500 , with the initial energy of 100 joules , simulation time of 100 seconds with the simulation area defined to be $2500 * 400$ sq.m. The proposed method is compared with the previous methods of the fuzzy shortest path for the adhoc networks [11] and the firefly based shortest path algorithm [9] to prove the heightened efficiency of the proposed method in terms of the successful message delivery, delay in transmission and the minimization in the energy consumption, that causes the improvement in the network lifetime of the network.

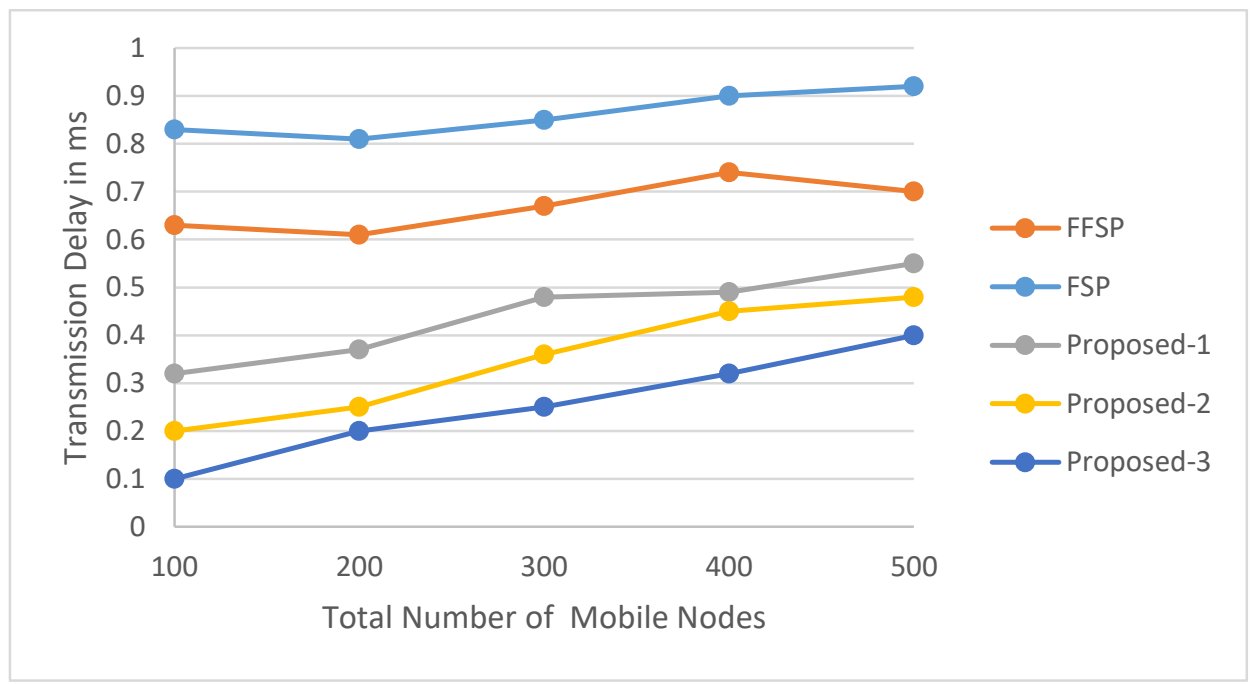

Fig .4 Delay in Transmission 
Journal of trends in Computer Science and Smart technology (TCSST) (2019)

Vol.01/No.01

Pages: 24-35

https://irojournals.com/tcsst/

DOI: https://doi.org/10.36548/jtcsst.2019.1.003

The Fig. 4 shows simulation result obtained for the delay in the transmission, the evaluation is done on the proposed method and the other two methods, fuzzy short path identification for the mobile adhoc networks and the firefly algorithm for the mobile networks in identifying the shortest path. The proposed method proves to have lesser delay compared to the prevailing, as the path established is stable, and faces negligible failures and rerouting that is caused by the path instability.

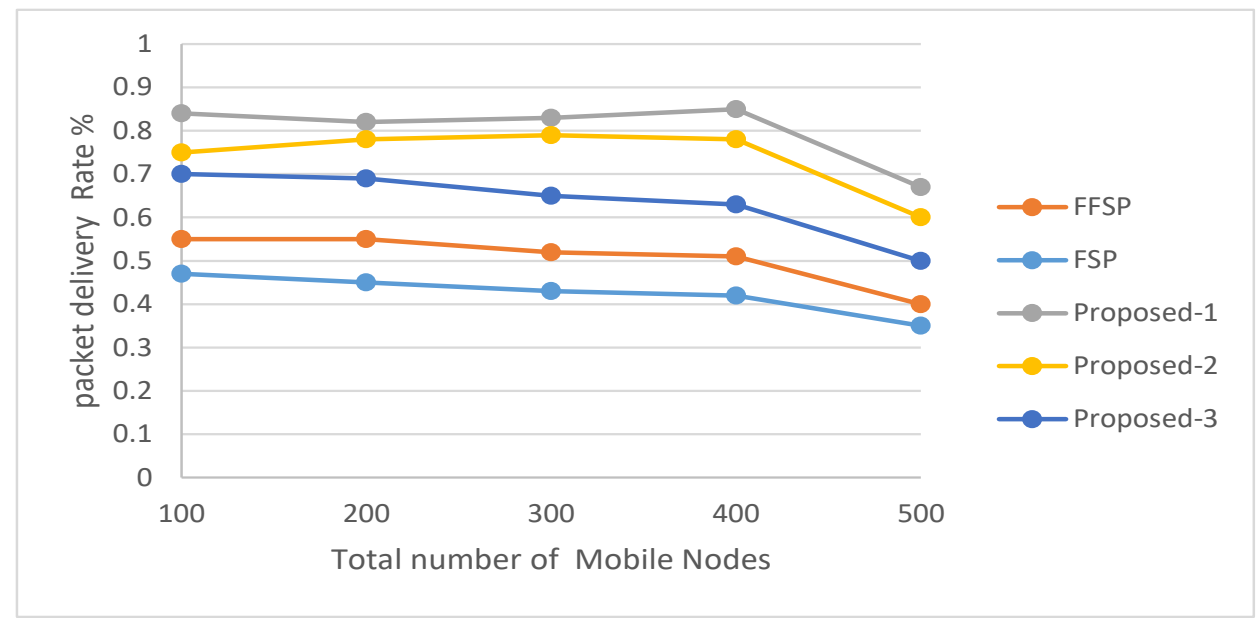

Fig.5Packet Delivery Rate

The fig. 5 shows the simulation result for the successful packet delivery, presenting the rate of the packets delivered by the proposed method and the prevailing method, the comparison highlights the proficiency of the proposed method as the packet delivery ratio of the reinforcement learning based stable short path establishment provides with the enhancement of $35 \%$ in the packet delivery rate compared to the fuzzy based short path (FSP) and $28 \%$ when compared with the firefly based short path establishment. 
Journal of trends in Computer Science and Smart technology (TCSST) (2019)

Vol.01/No.01

Pages: 24-35

https://irojournals.com/tcsst/

DOI: https://doi.org/10.36548/jtcsst.2019.1.003

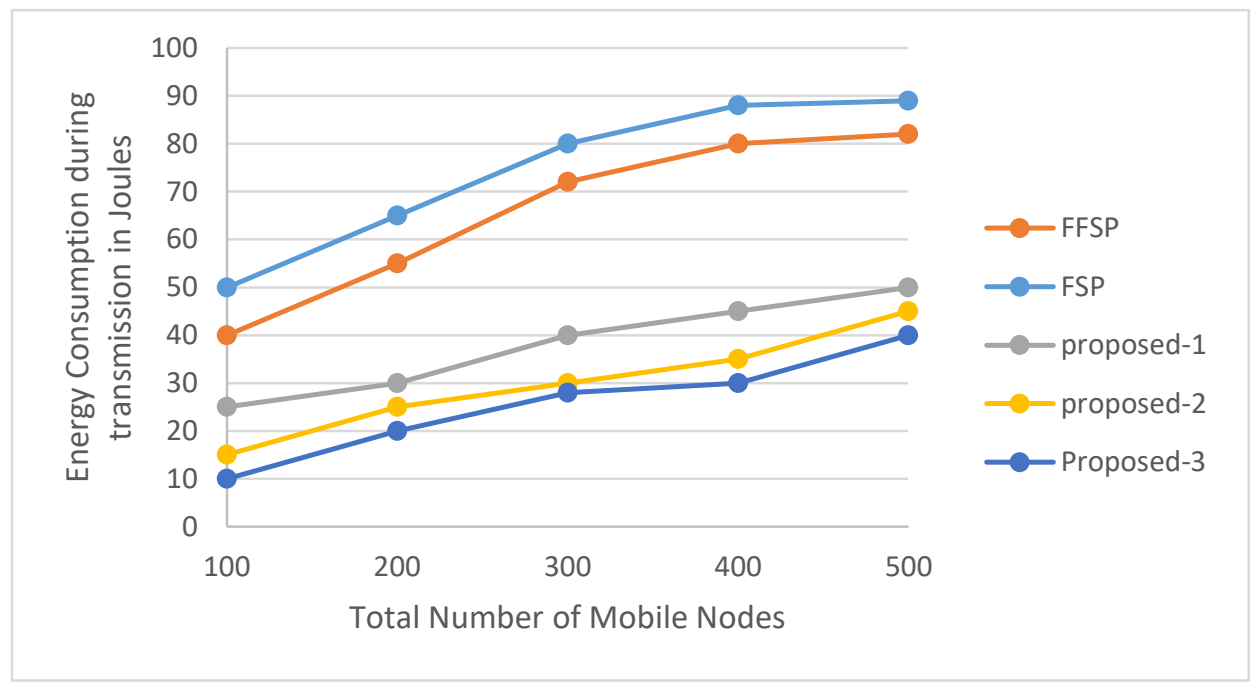

Fig .6 Energy Consumed During Transmission

The fig.6 gives the simulation result on the energy consumed for the process of transmission of the packets from the source to destination of the proposed method, and the prevailing method. Though all the three methods, have a limited energy consumption, due to the shortest path establishment from the source to destination, the proposed method proves to have a considerable amount of the energy saving due to stable connection established, eluding the circumstances of the rerouting and link failures.

\section{Conclusion}

The mobile adhoc network presenting complex challenges in the process of routing is addressed in the proposed method, utilizing the reinforcement learning. The method selects the node based on the delay in the transmission and the number of successful transmissions. The opts for the best group that has the highest reward on its actions for the transmission of the information and prefers with the neighboring node of very small distance to the destination as the next hop to establish as shortest path. Further the mobility prediction is done for each node, by framing a structure with the multiple states and assuming the mobile nodes to be in any one of the state, even on its movement and act according to operations done in the state. This is enables the reinforcement learning based short path establishment for the mobile adhoc networks to be stable. The performance evaluation on the grounds of the transmission delay, energy consumed during the transmission and the packet delivery ratio, and the comparison with the prevailing method show the proficiency of the proposed method over the prevailing. In future the paper is to proceed with the study on the upcoming enhancements to handle the security issues in the short path establishment for the mobile adhoc networks. 
Journal of trends in Computer Science and Smart technology (TCSST) (2019)

Vol.01/No.01

Pages: 24-35

https://irojournals.com/tcsst/

DOI: https://doi.org/10.36548/jtcsst.2019.1.003

\section{References}

1. Saeed, Nagham H., Maysam F. Abbod, and Hamed S. Al-Raweshidy. "MANET routing protocols taxonomy." In 2012 International Conference on Future Communication Networks, pp. 123-128. IEEE, 2012.

2. Bai, Yuxia, Yefa Mai, and Nan Wang. "Performance comparison and evaluation of the proactive and reactive routing protocols for MANETs." In 2017 Wireless Telecommunications Symposium (WTS), pp. 1-5. IEEE, 2017.

3. Nayak, Pad Malaya, and Pallavishree Sinha. "Analysis of random way point and random walk mobility model for reactive routing protocols for MANET using NetSim simulator." In 2015 3rd International Conference on Artificial Intelligence, Modelling and Simulation (AIMS), pp. 427-432. IEEE, 2015.

4. Er-Rouidi, Mohamed, Houda Moudni, Hicham Mouncif, and Abdelkrim Merbouha. "An energy consumption evaluation of reactive and proactive routing protocols in mobile ad-hoc network." In 2016 13th International Conference on Computer Graphics, Imaging and Visualization (CGiV), pp. 437-441. IEEE, 2016.

5. Darabkh, Khalid A., and Mohammad SE Judeh. "An Improved Reactive Routing Protocol over Mobile Adhoc Networks." In 2018 14th International Wireless Communications \& Mobile Computing Conference (IWCMC), pp. 707-711. IEEE, 2018.

6. Majd, Nahid Ebrahimi, Nam Ho, Thu Nguyen, and Jacob Stolmeier. "Evaluation of Parameters Affecting the Performance of Routing Protocols in Mobile Ad Hoc Networks (MANETs) with a Focus on Energy Efficiency." In Future of Information and Communication Conference, pp. 1210-1219. Springer, Cham, 2019.

7. Ghouti, Lahouari, Tarek R. Sheltami, and Khaled S. Alutaibi. "Mobility prediction in mobile ad hoc networks using extreme learning machines." Procedia Computer Science 19 (2013): 305-312.

8. Forster, Anna. "Machine learning techniques applied to wireless ad-hoc networks: Guide and survey." In 2007 3rd international conference on intelligent sensors, sensor networks and information, pp. 365-370. IEEE, 2007.

9. Darwish, Saad M., Amr Elmasry, and Shaymaa H. Ibrahim. "Optimal Shortest Path in Mobile Ad-Hoc Network Based on Fruit Fly Optimization Algorithm." In International Conference on Advanced Machine Learning Technologies and Applications, pp. 91-101. Springer, Cham, 2019.

10. Li, Fan, Xiaoyu Song, Huijie Chen, Xin Li, and Yu Wang. "Hierarchical Routing for Vehicular Ad Hoc Networks via Reinforcement Learning." IEEE Transactions on Vehicular Technology 68, no. 2 (2018): 18521865.

11. Ghasemnezhad, Solmaz, and Ali Ghaffari. "Fuzzy logic based reliable and real-time routing protocol for mobile ad hoc networks." Wireless Personal Communications 98, no. 1 (2018): 593-611. 
Journal of trends in Computer Science and Smart technology (TCSST) (2019)

Vol.01/No.01

Pages: 24-35

https://irojournals.com/tcsst/

DOI: https://doi.org/10.36548/jtcsst.2019.1.003

12. Thangaramya, K., K. Kulothungan, R. Logambigai, M. Selvi, Sannasi Ganapathy, and A. Kannan. "Energy aware cluster and neuro-fuzzy based routing algorithm for wireless sensor networks in IoT." Computer Networks 151 (2019): 211-223.

13. Nallusamy, C., and A. Sabari. "Particle Swarm Based Resource Optimized Geographic Routing for Improved Network Lifetime in MANET." Mobile Networks and Applications 24, no. 2 (2019): 375-385.

14. Kwon, Kiwoong, Seong Hoon Kim, Minkeun Ha, and Daeyoung Kim. "Traffic-aware stateless multipath routing for fault-tolerance in IEEE 802.15. 4 wireless mesh networks." Wireless Networks 24, no. 5 (2018): 1755-1774.

15. Sajwan, Mohit, Devashish Gosain, and Ajay K. Sharma. "Hybrid energy-efficient multi-path routing for wireless sensor networks." Computers \& Electrical Engineering 67 (2018): 96-113. 